\title{
Surface Effect on Mechanical Properties and Atomic Mobility of Ultrathin Polystyrene Films using Molecular Dynamics Simulations
}

\author{
Fan Yang ${ }^{\mathrm{a}}$, Zheng Zhong ${ }^{\mathrm{b}}$ \\ School of Aerospace Engineering and Applied Mechanics, 1239 Siping Rd., Shanghai, China, \\ 200092 \\ afanyang@tongji.edu.cn, bzhongk@tongji.edu.cn
}

Keywords: Surface effect, Polystyrene film, Molecular dynamics, Mechanical properties

\begin{abstract}
Thin polystyrene film has shown anomalous properties such as reduced glass transition temperature when the thickness is below $100 \mathrm{~nm}$. However, few attentions have been paid to its mechanical properties, which are important in understanding the stability and reliability of polymer nano-structures. In this work, we aim at elucidating the size dependent mechanical properties of ultrathin polystyrene films using molecular dynamics (MD) simulations. Coarse grained MD samples of free-standing PS films with different thicknesses were generated using the augmented phantom chain growth method. Active deformations were applied by moving two repulsive walls to determine the size dependent mechanical properties of the films. The distribution of local atomic mobility was investigated through partitioning the films into equidistant bins along thickness and calculating the mean square displacement for each bin. The results indicate the existence of a softened surface layer with reduced modulus and enhanced local atom mobility compared to the bulk state. It shows the deformation has an enhancing effect on the local atom mobility, especially along the thickness direction. This work can provide insights into the size dependent mechanical properties of ultrathin polymer films.
\end{abstract}

\section{Introduction}

Fast emerging nanoscale polymeric devices such as lab-on-a-chip and drug delivery systems [1] have shown vast potential of applications in medicine, energy, etc., and have led to wide-spread research on the properties of polymer materials at nanoscale. Considerable attentions have been paid to the anomalous properties of polystyrene (PS) thin films with thickness below 100 nanometers, such as lower density, larger free volume and reduced glass transition temperature Tg [2-4]. For the mechanical properties, several experimental [5,6] and numerical $[7,8]$ efforts have been made. It is found that ultrathin PS films show softened response compared to bulk as the thickness decreases below 30 or $40 \mathrm{~nm}$. However, researches on the mechanical properties and the underlining mechanisms are still not sufficient for the ultrathin PS films, especially with respect to variation along the thickness direction.

The size dependent properties of ultrathin PS films are believed to come from the effect of configurational constraints at the surface and interface [9-11]. Disagreement in the explanation of size dependence still exists on whether there is a liquid like layer near the surface [12,13]. Experimental techniques utilizing nanoindentation [5,14], nanoparticle embedding [15,16] and instable wrinkling $[6,17]$ have been developed to explore the mechanical properties of ultrathin films and coatings. However, these techniques cannot evade the far field effects from the substrate and the indenter 5 since these experiments can only be carried out on the substrate-supporting specimens. Thus determining the surface effect of very thin polymer films from experiments is a considerable challenge. MD simulations, on the other hand, can be useful in understanding the size dependent properties of polymeric nanostructures from the bottom-up viewpoint. A number of researches $[8,18,19]$ have been conducted using MD to investigate the polymer thin films, and obtained useful results. 
In this paper, we aim at investigating the variation of local mechanical properties of ultrathin PS films along thickness direction using molecular dynamics simulations. The local atomic mobility is also investigated in order to provide insight into the origin of the size dependent properties.

\section{Model of PS Films under Compressing Deformation}

A series of computational samples are created for PS thin films with different thicknesses using the augmented PCG scheme [20]. Periodic boundaries are applied for the two lateral directions and finite boundaries are applied for the thickness direction. For comparison, a bulk sample is also created with periodic boundaries along all three directions. The coarse grained representation that incorporates $\mathrm{H}$ atoms into the CHx united particles, is applied to model the PS macromolecules. The force field for PS consists of bonded and non-bonded interactions, with total potential energy written as:

$$
U=\frac{1}{2} k_{B}\left(r-r_{0}\right)^{2}+\frac{1}{2} k_{\theta}\left(\theta-\theta_{0}\right)^{2}+\frac{1}{2} \sum_{j=1}^{3} k_{\phi}^{j} \cos \left[1+(-1)^{j+1} j \phi\right]+\frac{1}{2} k_{\psi}\left(\psi-\psi_{0}\right)^{2}+4 \varepsilon\left[\left(\frac{\sigma}{r}\right)^{12}-\left(\frac{\sigma}{r}\right)^{6}\right] \text {. }
$$

Each item on the right hand side corresponds respectively to the stretch, angle, improper and dihedral energy for bonded interaction and the LJ energy for non-bonded interaction. The meanings of the variables and the values of the constant parameters can be found in the work by Srivastava et al. (2010). A cutoff distance of $12 \AA$ is used for the non-bonded interactions.

After initial generation, the samples are firstly relaxed in NVE ensemble with a limit on the atom displacement in each time step to push apart atoms that are unrealistically too close. The samples are then annealed at $600 \mathrm{~K}$ for a time of $0.5 \mathrm{~ns}$ and cooled to $300 \mathrm{~K}$ over another $0.5 \mathrm{~ns}$. After that the system is further relaxed in NPT ensemble at a thermostat of $300 \mathrm{~K}$ with $100 \mathrm{fs}$ relaxation time and a barostat of one atmosphere with 200 fs relaxation time. For the film sample, only the stress components along the lateral directions are controlled and the stress along the thickness direction was self-equilibrated. These pre-processing procedures ensure the atoms in the samples are initially equilibrated. The created film samples is $75 \pm 3 \AA$ in lateral size, with varying thickness depending on the number of PS chains contained in the sample. Fig. 1 shows the obtained five film samples with thicknesses 49, 69, 118, 177 and $228 \AA$, containing 5, 10, 20, 30, and 40 PS chains respectively. Each chain has 200 monomers, rendering a molecular weight MW $=21 \mathrm{k}$.
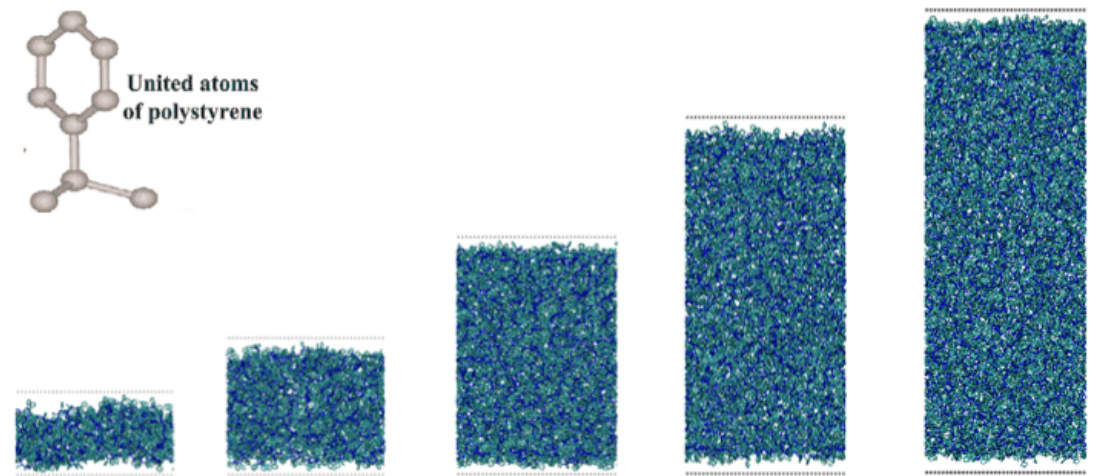

Fig. 1 Configurations of the MD film samples with different thicknesses

The simulations are carried out using the general-purpose MD package LAMMPS. All the deformations are carried out in NVT ensemble at temperature of $300 \mathrm{~K}$. The equations of motion are integrated by velocity Verlet algorithm with a time step of 2 fs. For the film samples, two repulsive walls are included at the top and bottom of the sample to impose the compressing deformation. The repulsive interactions between the wall and the PS atoms are given by a LJ typed potential following the work of Yang et al. The walls are initially located at the positions where the resultant force on the wall changes from zero to nonzero. The deformation is applied by simultaneously moving the repulsive walls through function "fix wall/lj126" of LAMMPS. For the bulk sample, the deformation is implemented by affinely altering the atomic coordinates and/or velocities at regular intervals, 
utilizing the LAMMPS function "fix deform". It is pointed out that the velocity also needs to be updated for the atoms crossing the periodic boundaries for correct energy calculation [21]. This consideration is also implemented in this work.

\section{Results and Discussions}

Size Dependent Mechanical Properties. Simple deformation of uniaxial straining along $\mathrm{z}$-axis/thickness direction is applied to the obtained PS samples, with the two lateral directions ( $\mathrm{x}$ and y) held fixed. The engineering strain rate is kept as $1 \times 10^{9} \mathrm{~s}^{-1}$ for all samples. That means the repulsive walls are moved at different velocities for different thickness films. According to the discussions on stress definition in MD [21], the averaged virial stress over the entire simulation specimen is used as the Cauchy stress. For the film case the stress calculated in this way agrees well with that calculated from the force on the repulsive walls.

The stress-strain responses for films of different thicknesses are plotted in Fig. 2, compared with the bulk case. In this graph, the values of stresses and strains in figures refer to their magnitudes. It shows an evident decrease in the slope of the stress-strain curve as the film thickness decreases, with the bulk case as the upper bound. The results indicate a considerable reduction of stiffness for the thinner film. It is also noted that the unloading curve almost coincides with the nonlinear loading curve at the engineering strain of 0.3 for all samples, signifying the hyperelastic properties of the PS material.

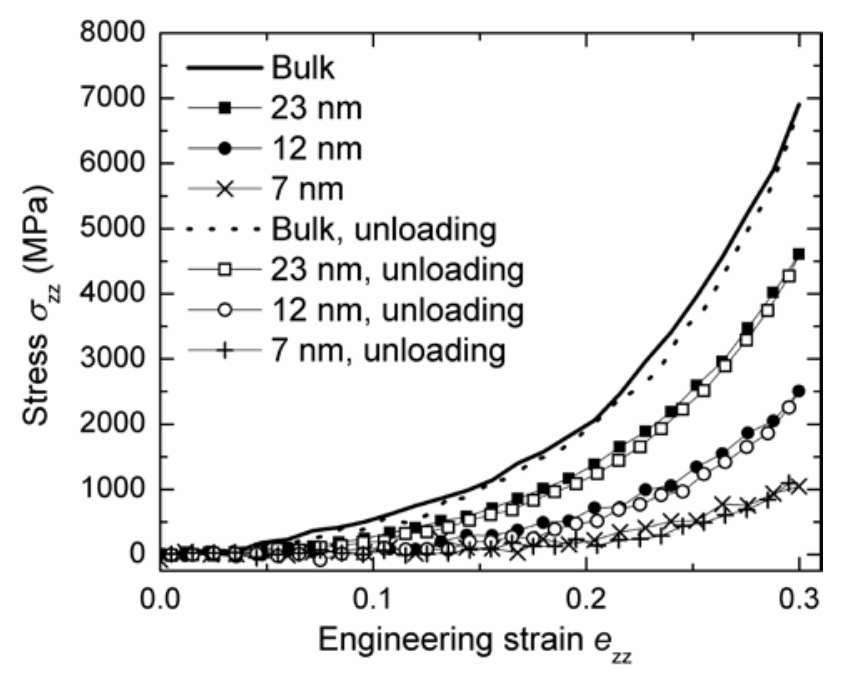

Fig. 2 stress-strain curves for both the bulk and film samples with different thicknesses

Variation of Mechanical Properties along Film Thickness. In order to investigate the variation of local properties along film thickness direction, the film is partitioned into a number of bins with equal intervals along the thickness. The apparent displacement of each bin is calculated by averaging the displacements of all the atoms whose original positions are in that bin. The local strain is then calculated based on the displacements of the bins. This calculation ignores the effect of atom diffusion across the boundaries of adjacent bins. Since the simulation time of $300 \mathrm{ps}$ is short for the mass diffusion, this treatment is considered an acceptable simplification. Fig. 3a and $3 \mathrm{~b}$ show the variations of the local displacement and strain along film thickness for different samples at the overall engineering strain 0.3 respectively. It shows that the strains near the surfaces are obviously larger than at the core, indicating that the PS thin films can be considered to be composed of relatively softer surface layers and a harder core region. From Fig. 3b, the depth of surface region with higher strain is comparable for different thickness films, thus leading to a larger ratio of surface region for the thinner film. This can explain the reduced stiffness of the thin films when the thickness is decreased. 


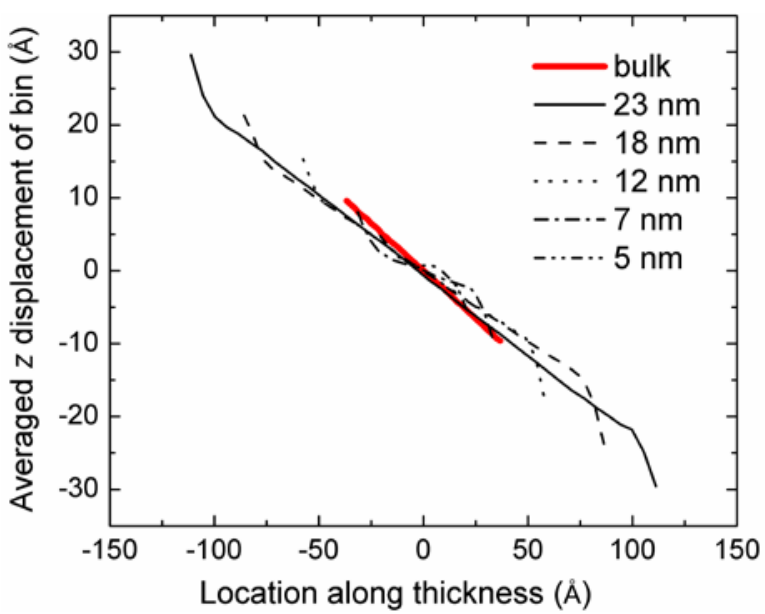

(a)

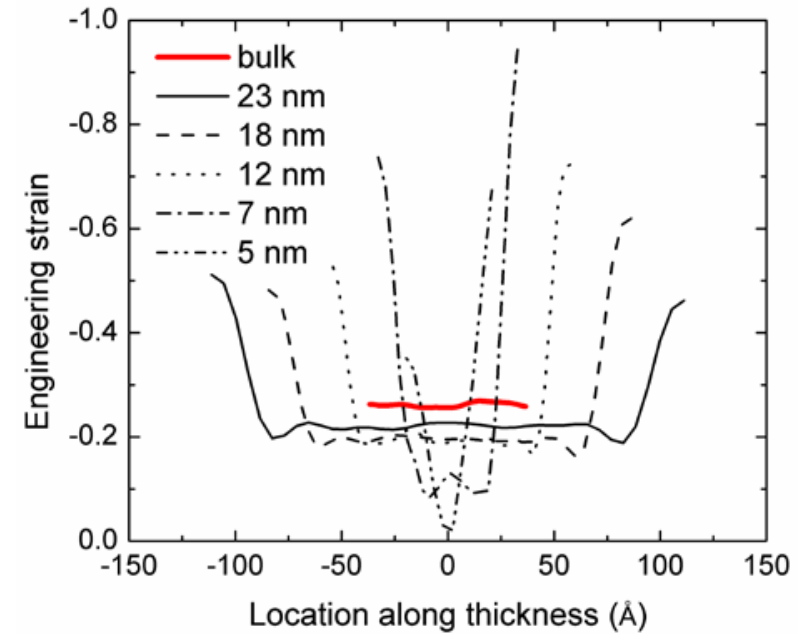

(b)

Fig. 3 Variation of (a) displacement and (b) strain along z/thickness direction for different samples

Variation of Local Chain Mobility along Film Thickness. Macro properties of a material are always rooted in its microstructures. The size dependent mechanical properties of thin PS films are believed to come from the less configurational constraints at the surface and the resulted effect on atomic mobility. As stated in the literature $[18,19]$, the near surface region has a lower density and a larger free volume, allowing for larger atomic mobility compared to the bulk region. In this section, we investigate the variation of local chain mobility along film thickness through the mean square displacement (MSD) and its components, especially under the deformation condition. The MSD and its components are defined as

$$
\begin{aligned}
\operatorname{MSD} & =\left\langle|\mathbf{x}(t)-\mathbf{x}(0)|^{2}\right\rangle=\frac{1}{N} \sum_{k=1}^{N}\left|\mathbf{x}^{(k)}(t)-\mathbf{x}^{(k)}(0)\right|^{2} \\
\operatorname{MSD}_{i} & =\left\langle\left(x_{i}(t)-x_{i}(0)\right)^{2}\right\rangle=\frac{1}{N} \sum_{k=1}^{N}\left(x_{i}^{(k)}(t)-x_{i}^{(k)}(0)\right)^{2}
\end{aligned}
$$

where, $\mathbf{x}^{(k)}$ is the displacement vector of atom $k . x_{i}^{(k)}$ is the displacement component along the $i^{\text {th }}$ direction. $i=1,2,3$ corresponding to $\mathrm{x}, \mathrm{y}$ and $\mathrm{z}$ axis respectively. For the deformation case, the convective displacement due to the deformation is irrelevant to the local chain motion, and should be subtracted from the overall displacement for each atom. Thus the equations for calculating MSD and its components for a film under compression along z-axis direction are:

$$
\begin{aligned}
\operatorname{MSD} & =\frac{1}{N} \sum_{k=1}^{N}\left|\mathbf{x}^{(k)}(t)-\mathbf{x}^{(k)}(0)-\varepsilon_{z z}(t) z^{(k)}(0) \mathbf{k}\right|^{2} \\
\operatorname{MSD}_{i} & =\frac{1}{N} \sum_{k=1}^{N}\left(x_{i}^{(k)}(t)-x_{i}^{(k)}(0)-\varepsilon_{z z}(t) z^{(k)}(0) \delta_{i 3}\right)^{2}
\end{aligned}
$$

where, $\varepsilon_{z z}(t)$ is the engineering strain at time $t, \mathbf{k}$ is the unit vector along z-axis, $\delta_{i j}=1$ only when $i$ $=j$. In order to obtain the spatial variation of MSD, the film is partitioned into a number of bins along the thickness and the MSD is averaged for each bin based on the atoms whose initial positions are in that bin. This algorithm ignores the possibility for the atoms leaving the original bin and entering other bins. Given the low mobility of glassy PS and the short time analyzed here, this possibility does not cause serious problems.

Fig. 4a and 4b show MSD evolution of different bins from surface/boundary to center for deforming the $23 \mathrm{~nm}$ thick film and the bulk samples, respectively. Similar to the un-deformed case as stated in literature [18], the atoms near the surface have a larger MSD, thus a larger mobility for the 
film under deformation. On the other hand, Fig. 4b shows that the MSD does not differ much from boundary to the center for the bulk sample.

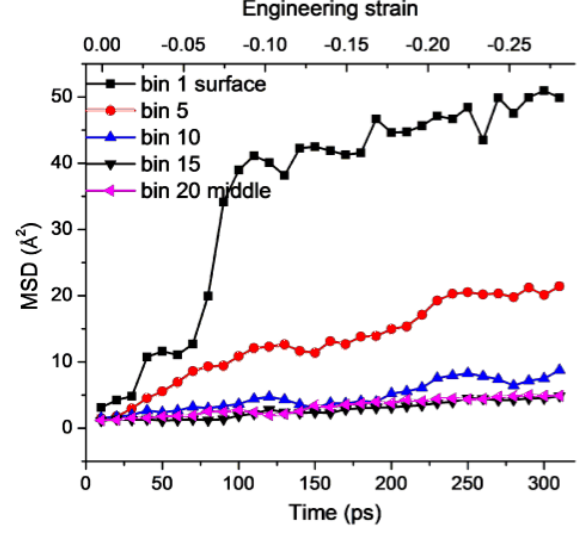

(a)
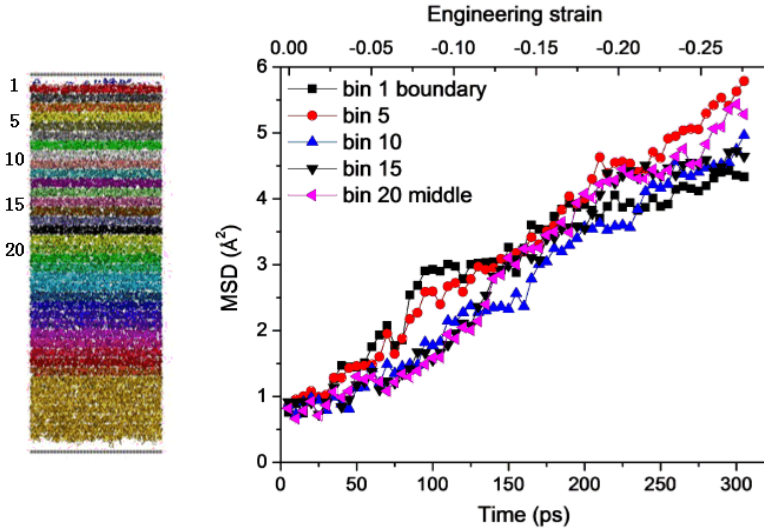

(b)

Fig. 4 Variation of MSD along thickness/z direction for (a) film and (b) bulk samples

Fig. 5 compares the distributions of the three MSD components in the deformed film with those in the un-deformed film. It shows that the application of deformation can largely enhance the MSD of the film, especially along the thickness direction. The decrease of MSD in the region very near the surface can be attributed to the constraint effect from the repulsive walls. The results indicate an enhancing effect of deformation application on the mobility of the PS atoms. Furthermore, the atomic motion becomes anisotropic under the deformation, with more mobility along the deforming direction.

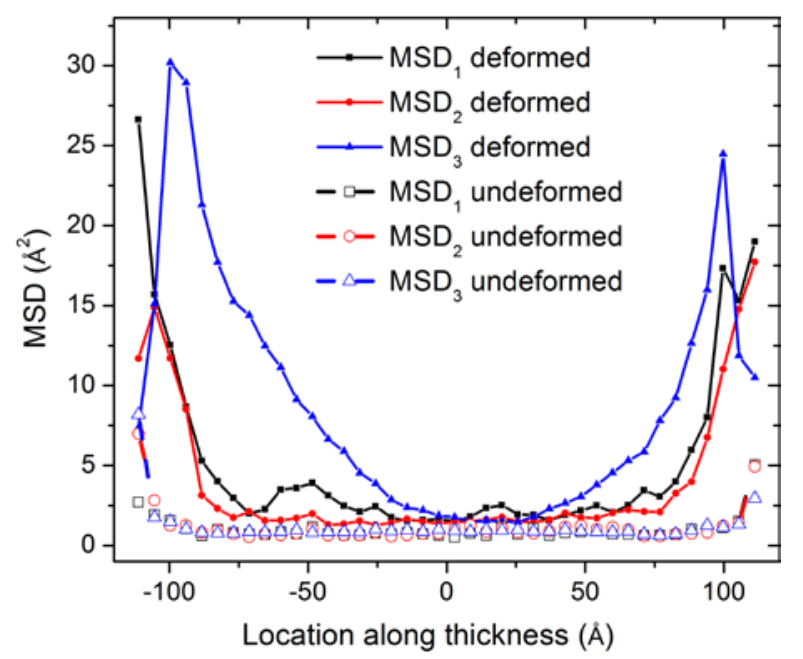

Fig. 5 Comparison of the MSD distributions in the deformed film with those in the un-deformed film

\section{Conclusions}

In this paper the size dependent mechanical properties of thin PS films are investigated using MD simulations. The variations of local mechanical properties and local chain mobility along the film thickness are determined via partitioning the film into a number of bins along thickness and averaging the properties for each bin. The results indicate the existence of a softened layer near the film surface with reduced stiffness and larger atomic mobility. The effect of deformation application on the atomic mobility is also investigated and an enhancing effect is found for the deformation on the PS atom mobility, especially along the deforming direction.

\section{Acknowledgements}

We acknowledge the support from National Natural Science Foundation of China (11402173), Innovation Program of Shanghai Municipal Education Commission (15zz018), the Fundamental 
Research Funds for the Central Universities (20143401; 1500219095), and the Shanghai Supercomputer Center.

\section{References}

[1] Y. Yang, D. Liu, Y. Xie, J. Lee and D. Tomasko: Adv. Mater. Vol. 19 (2007), p. 251.

[2] C. Ellison and J. Torkelson: Nat. Mater. Vol. 2 (2003), p. 695.

[3] Z. Fakhraai and J. Forrest: Science Vol. 319 (2008), p. 600.

[4] J. Sharp, J. Teichroeb and J. Forrest: Eur. Phys. J. E Vol. 15 (2004), p. 473.

[5] K. Miyake, N. Satomi and S. Sasaki: Appl. Phys. Lett. Vol. 89 (2006), p. 31925.

[6] J. Torres, C. Stafford and B. Vogt: Acs Nano Vol. 3 (2009), p. 2677.

[7] T. Bohme and J. de Pablo: J. Chem. Phys. Vol. 116 (2002), p. 9939.

[8] K. Yoshimoto, T. Jain, P. Nealey and J. de Pablo: J. Chem. Phys. Vol. 122 (2005), p. 144712.

[9] J. Kim, J. Jang and W. Zin: Langmuir Vol. 17 (2001), p. 2703.

[10]R. Priestley, C. Ellison and L. Broadbelt: Science Vol. 309 (2005), p. 456.

[11]J. Sharp and J. Forrest: Phys. Rev. Lett. Vol. 91 (2003), p. 235701.

[12] S. Hutcheson and G. McKenna: Eur. Phys. J. E Vol. 22 (2007), p. 281.

[13] J. Sharp, J. Forrest, Z. Fakhraai, M. Khomenko, J. Teichroeb and K. Dalnoki-Veress: J. E Vol. 22 (2007), p. 287.

[14]J. Zhou and K. Komvopoulos: J. Appl. Phys. Vol. 100 (2006), p. 114329.

[15]J. Erichsen, J. Kanzow, U. Schurmann, K. Dolgner, K. Gunther-Schade, T. Strunskus, V. Zaporojtchenko and F. Faupel: Macromolecules Vol. 37 (2004), p. 1831.

[16] J. Yang, C. Liu, Y. Yang, B. Zhu, L. Lee, H. Chen and Y. Jean: J. Polym. Sci. B: Polym Phys. Vol. 47 (2009), p. 1535.

[17]C. Stafford, B. Vogt, C. Harrison, D. Julthongpiput and R. Huang: Macromolecules Vol. 39 (2006), p. 5095.

[18]K. Mansfield and D. Theodorou: Macromolecules Vol. 24 (1991), p. 6283.

[19]F. Yang, S. Ghosh and J. Lee: Comput. Mech. Vol. 50 (2012), p. 169.

[20]A. Srivastava, C. Alleman, S. Ghosh and J. Lee: Modelling Simul. Mater. Sci. Eng. Vol. 18 (2010), p. 065003.

[21]F. Yang and Z. Zhong: J. Mech. Phys. Solids Vol. 77 (2015), p. 146. 